\title{
ATRIBUTOS POLARIMÉTRICOS DE IMAGEM RADAR NA INFERÊNCIA DE PARÂMETROS MORFOLÓGICOS DE MACRÓFITAS
}

\author{
Radar image polarimetric attributes for the inference of macrophyte morphologic \\ parameters \\ LAURIANA RÚBIO SARTORI ${ }^{1}$ \\ NILTON NOBUHIRO IMAI ${ }^{1}$ \\ JOSÉ CLÁUDIO MURA ${ }^{2}$ \\ VILMA MAYUMI TACHIBANA ${ }^{1}$ \\ ${ }^{1}$ Universidade Estadual Paulista \\ Programa de Pós-Graduação em Ciências Cartográficas \\ Rua Roberto Simonsen, 305 \\ CEP: 19060-900 - Presidente Prudente, SP. \\ ${ }^{2}$ Instituto Nacional de Pesquisas Espaciais \\ Departamento de Processamento de Imagens (DPI) \\ Rua dos Astronautas, 1758 \\ CEP: 12227-010 São José dos Campos, SP. \\ lauriunesp@gmail.com; nnimai@fct.unesp.br; mura@dpi.inpe.br; \\ vilma@fct.unesp.br
}

\section{RESUMO}

O propósito deste trabalho é ajustar um modelo de regressão múltipla em função de atributos de imagens de radar polarimétrico, para estimativa da variável morfológica "volume da haste" de macrófitas encontradas na planície de inundação da Amazônia, no Lago Grande de Monte Alegre (Pará - Brasil). Com esta estimativa, pretende-se avaliar o potencial dos dados polarimétricos do sensor ALOS/PALSAR, destacando a importância da informação de fase das imagens de radar. Para este estudo, foram coletados dados de campo em época próxima à aquisição do dado de radar. Variáveis morfológicas, como altura e diâmetro da haste emergente, de três espécies de macrófitas foram medidas em campo e usadas para derivar o "volume da haste", o qual foi modelado usando atributos da imagem de radar. Dois atributos da decomposição de Freeman-Durden, dois de Touzi e um de Pope apresentaram melhor desempenho na modelagem. Apesar do modelo não ter obtido um elevado 
coeficiente de determinação (44\%), ele apresentou boa capacidade preditiva, já que todos os elementos de validação caíram dentro do intervalo de predição de $95 \%$ de confiança. Dentre as cinco variáveis independentes do modelo, quatro foram geradas a partir da informação de fase das imagens, o que reforça a importância dessa informação.

Palavras-chave: Radar Polarimétrico; PALSAR; Regressão Linear Múltipla; Monitoramento de Macrófitas; Planície de Inundação da Amazônia.

\section{ABSTRACT}

This work aims at modelling the morphological variable steam-volume of the macrophyte species as a function of the attributes derived from the ALOS/PALSAR polarimetric data using multiple regression technique. The study was carried out at Monte Alegre Lake, in the Amazon floodplain area, Pará State, Brazil. The modeled variable steam-volume was evaluated and the contribution of the phase information from the radar data was highlighted. The fieldwork was performed almost simultaneously to the radar acquisition. Macrophyte morphological variables were measured in the field and used to derive the stem-volume model regarding the attributes generated from the radar data. Although the adjusted coefficient of determination was not high $\left(R_{a j}^{2}=44 \%\right)$, the presented predictive ability and all validation elements were expected with $95 \%$ of confidence. Among the five independent variables of the model, four were generated from the phase information, which brings about this reliable information.

Keywords: Polarimetric Radar; PALSAR data; Multiple Linear Regression; Macrophyte Monitoring; Amazon Floodplain.

\section{INTRODUÇÃO}

Uma imagem de radar é uma matriz bidimensional de pixels onde cada pixel é formado por um número complexo (amplitude e informação de fase) associado à refletividade dos espalhadores contidos dentro da célula de resolução do radar (LEE e POTTIER, 2009).

Dados polarimétricos são aqueles que registram a informação completa do espalhamento do radar, incluindo tanto a amplitude quanto a informação de fase dos quatro canais de polarização (HH, HV, VH e VV). O canal de polarização HV, por exemplo, significa que o sinal foi transmitido por uma antena de polarização horizontal $(h)$ e recebido por uma antena de polarização vertical $(v)$.

Dados de sensoriamento remoto por radar são muito utilizados em áreas alagáveis (POPE et al., 1994; HESS et al., 1995; COSTA et al., 2005; NOVO et al., 2002; HESS et al. 2003; TOUZI et al., 2007) por serem de difícil acesso e por possuírem alta freqüência de cobertura de nuvens, o que dificulta o uso de sensores ópticos (POPE et al., 1994). 
Universalmente conhecidas como "wetlands", as áreas alagáveis são regiões que oscilam periodicamente entre fases terrestres e aquáticas. Entre os alvos presentes nas áreas alagáveis, o papel da vegetação aquática herbácea (macrófita) é único e importante. Elas são responsáveis por grande parte da biodiversidade das áreas alagáveis (MITSCH e GOSSELINK, 2000) e desempenham diferentes funções ecológicas (THOMAZ e BINI, 2003). Em contrapartida, são habitats favoráveis à reprodução de vetores de doenças de veiculação hídrica (malária, dengue, febre amarela, esquistossomose) com sérias conseqüências para a saúde pública (PEDRALLI, 2003; VASCONCELOS et al., 2006). Respondem pelas maiores taxas de emissão de metano quando comparadas às áreas de floresta inundada ou águas abertas (DEVOL et al., 1988; BARTLET et al., 1990).

Os estudos das macrófitas utilizando-se dados de radar foram feitos no sentido de se investigar suas propriedades biofísicas, sua distribuição e sua produtividade (HESS, et al., 1995; HESS et al., 2003; COSTA, 2005; SILVA, et al., 2010). Alguns estudos usaram apenas a informação de amplitude das imagens; outros, as informações das imagens polarimétricas de radar aerotransportado. Com a recente disponibilidade de dados SAR polarimétricos orbitais, torna-se oportuna a investigação a respeito da importância da informação da fase para o estudo de macrófitas.

Supondo-se que seja possível inferir variáveis morfológicas de uma planta aquática emersa em função de atributos de radar, este trabalho tem como objetivo ajustar e avaliar um modelo de regressão múltipla em função de atributos da imagem SAR polarimétrica para estimativa da variável morfológica "volume da haste". A partir dessa estimativa, pode-se avaliar o potencial do uso de imagem SAR polarimétrica na banda L (ALOS/PALSAR), mais especificamente o que se refere à informação da fase para a referida estimativa.

\section{2. ÁREA DE ESTUDOS}

A área de estudo é a planície de inundação do Lago Grande de Monte Alegre (Pará, Brasil), próximo à cidade de Santarém, entre as coordenadas $803265 \mathrm{~m} \mathrm{E} /$ 9751336 m N a 827317 m E / 9776919 m N, projeção UTM (Universal Transverse Mercator), zona 21 sul, sistema de referência WGS 84.

O padrão de inundação na área de estudo compreende um pulso de inundação anual, único, com o aumento da lâmina d'água de dezembro até junho e o recuo de julho a dezembro. As variações diárias no nível da água são pequenas, exceto durante os pontos de transição em junho-julho e novembro-dezembro.

\section{AQUISIÇÃO DA IMAGEM PALSAR E DOS DADOS DE MACRÓFITAS}

A imagem PALSAR foi adquirida dia 25 de março de 2009, no modo PLR (polarimétrico), com ângulo off-nadir de $21,5^{\circ}$, órbita ascendente e nível de processamento 1.1 (dado SLC - Single Look Complex). O espaçamento em pixel e linha da imagem adquirida é, respectivamente, 9,369 e 3,574 m. 
A coleta de dados em campo foi realizada nas datas mais próximas possíveis da aquisição da imagem (Abril/2009) para medir variáveis morfológicas, como altura da haste emergente ( $\mathrm{L}_{\text {haste }}$ ) das macrófitas e diâmetro da haste emergente $\left(\right.$ Diam $\left._{\text {haste }}\right)$. A partir destas duas variáveis, foi derivado o "volume da haste" emergente $\left(\mathrm{V}_{\text {haste }}\right)$, o qual foi determinado como um volume cilíndrico ( $\mathrm{V}_{\text {haste }}=\mathrm{L}_{\text {haste }}$ x 0,25 x $\pi \times$ Diam $_{\text {haste }}^{2}$ ), conforme Silva et al., (2009).

No total, 65 elementos amostrais foram adquiridos: 41 Paspalum repens P.J.Bergius (PR), 15 Hymenachne amplexicaulis (Rudge) Nees (HA) e 9 Panicum elephantipes Nees (PE).

\subsection{Processamento da imagem PALSAR e dos elementos amostrais}

A partir do dado PALSAR, modo SLC, foi obtida a matriz de espalhamento, a qual foi convertida na matriz de covariância com uma média espacial de $7 \times 1$ pixels (azimute $\mathrm{x}$ range, respectivamente). A fim de reduzir o ruído speckle, a matriz de covariância foi filtrada usando o filtro de Lee, com janela 3x3 pixels (LEE, 1981). Esta escolha foi feita, pois testes realizados com janelas maiores suavizaram excessivamente a cena.

A partir da imagem filtrada, os seguintes atributos radar foram extraídos a partir dos dados de amplitude: sigma zero para as polarizações $\mathrm{HH}, \mathrm{HV}$ e VV (HENDERSON e LEWIS, 1998), span, razão de co-polarização (razão entre as polarizações paralelas HH e VV) e polarização cruzada (razão entre a polarização paralela HH ou VV e a polarização cruzada HV), parâmetros de Pope (índice de biomassa, índice de estrutura da copa e índice do espalhamento do volume) (POPE et al., 1994). Os atributos extraídos a partir da amplitude em conjunto com a informação de fase foram: parâmetros da decomposição de Cloude-Pottier (entropia, ângulo alfa e anisotropia) (CLOUDE e POTTIER, 1996), de Freeman-Durden (espalhamento volumétrico, espalhamento double-bounce e espalhamento superficial) (FREEMAN e DURDEN, 1998) e de Touzi (magnitude do tipo de espalhamento, fase do tipo de espalhamento, helicidade, ângulo de orientação) (TOUZI, 2007), a diferença de fase entre HH e VV e a magnitude do coeficiente de correlação cruzada. Cada parâmetro de Touzi foi gerado para o primeiro, segundo, terceiro mecanismo de espalhamento e para média deles. No total, 34 atributos foram extraídos. Maiores detalhes sobre o processamento da imagem PALSAR e a geração de atributos podem ser vistos em Sartori et al. (2011).

Para cada elemento amostral $(50 \mathrm{~m} \times 50 \mathrm{~m})$ na imagem radar, a média foi computada sobre cada atributo extraído. Foi especificada esta área quadrada de 50 $\mathrm{m} \times 50 \mathrm{~m}$, pois corresponde ao tamanho médio de bancos de macrófitas homogêneos observados em campo.

\section{MODELAGEM DA VARIÁVEL MORFOLÓGICA EM FUNÇÃO DOS ATRIBUTOS POLARIMÉTRICOS}

Para realizar a análise de regressão múltipla foram utilizados 65 elementos amostrais, sendo que destes, 55 elementos (35 de PR, 13 de HA e 7 de PE) foram 
separados para a estimativa do modelo e 10 (6 de PR, 2 de HA e 2 de PE) para a validação.

O seguinte conjunto de etapas adotadas por Narvaes (2010) e Gonçalves et al. (2011) foi também utilizado neste trabalho para a modelagem da variável morfológica em função dos atributos de radar:

1. Análise exploratória. A partir desse tipo de investigação foram identificados os atributos de radar com maior capacidade para explicar o modelo, verificando-se ainda a existência da relação linear entre as variáveis independentes e a dependente.

2. Seleção das variáveis explicativas. O subconjunto de atributos adequado à modelagem foi selecionado utilizando o algoritmo "Best subsets", o qual procura, automaticamente, pelos melhores subconjuntos segundo um dado critério. A seleção do subconjunto foi baseada no coeficiente de determinação ajustado $\left(R_{a j}^{2}\right)$ e no erro médio quadrático ( $E M Q)$, plotados contra as $(p-1)$ variáveis explicativas.

3. Diagnósticos. Para verificar se o modelo atende as suposições exigidas, foram feitas as seguintes verificações:

a) Normalidade da variável dependente através do teste Shapiro-Wilk (SHAPIRO et al., 1968) ao nível de significância de 5\%. Quando necessário foi aplicada a transformação Box-Cox para que a variável tivesse distribuição normal.

b) Análise do gráfico de dispersão de cada variável explicativa versus a variável dependente, com finalidade de entender o comportamento entre elas;

c) Normalidade e homocedasticidade dos resíduos, através do teste de Levene modificado, ao nível de significância de 5\%.

d) Outliers influentes: avaliados pela distância Cook e DFFITS;

e) Termos de interação, em cujo procedimento os resíduos do modelo de regressão foram plotados contra cada termo de interação e analisada, por análise visual, a ocorrência de padrão sistemático. Caso houvesse, havia necessidade de inclusão do termo de interação no modelo;

f) Multicolinearidade, detectada pelo fator de inflação da variação (VIF) que, se superior a 10 indica que a multicolinearidade pode estar influenciando indevidamente as estimativas dos mínimos quadrados (NETER et al., 2004);

g) Autocorrelação espacial, avaliada pelo diagrama de espalhamento de Moran (ANSELIN, 1996).

4. Validação do modelo. Foi utilizado o critério PRESS (soma dos quadrados dos erros de predição - prediction error sum of squares), o qual é uma medida que indica quão bom é o modelo de regressão na predição de novos dados. Para o cálculo do PRESS, é excluída a i-ésima observação e estimada a função de regressão para os (n-1) casos restantes. A partir desta estimativa, é predito o 
valor da observação excluída. Este procedimento é repetido para as $n$ observações, sendo feita a exclusão de uma observação de cada vez. O PRESS é dado pela soma quadrática dos $n$ erros de predição. $O$ resultado do cálculo do PRESS é comparado ao do SQR do modelo (em que as n observações são consideradas). Segundo Neter et al. (2004), se o PRESS for próximo de SQR (soma dos quadrados dos resíduos), o MSE (mean square error) pode ser considerado um indicador da capacidade preditiva do modelo, ou seja, um estimador do desvio padrão. Além do critério PRESS foi utilizado um conjunto de observações reservadas para a validação. Os valores observados foram comparados aos valores preditos apresentados com o intervalo de $95 \%$ de confiança.

Figura 1 - Fluxograma adotado para a modelagem da variável morfológica.

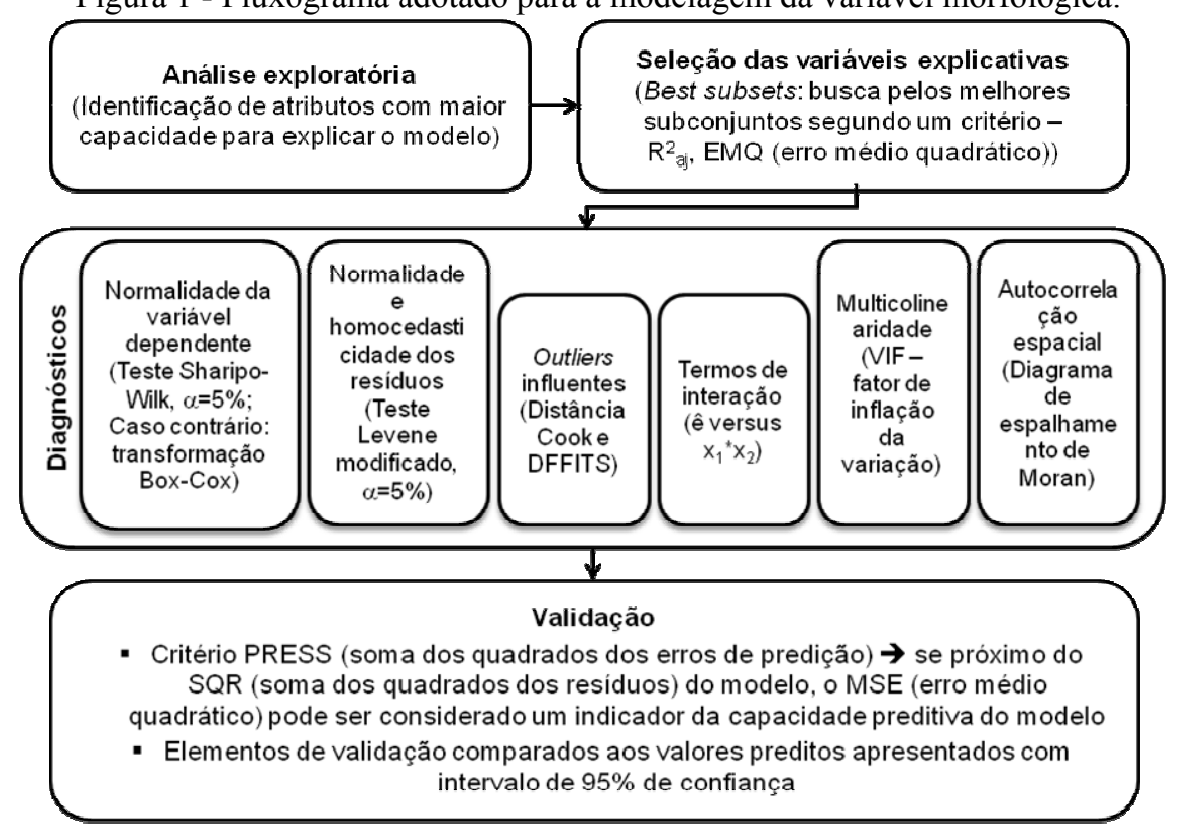

\section{RESULTADOS E DISCUSSÕES}

Os resultados, conforme o conjunto de etapas metodológicas apresentadas foram:

1. Análise exploratória. A variável "espalhamento volumétrico", derivada da decomposição de Freeman-Durden, possui uma das mais altas correlações $(0,55)$ com a variável morfológica "volume da haste" e por isso decidiu-se mantê-la no modelo; 
2. Seleção das variáveis explicativas. A Figura 2 mostra o gráfico de $R_{a j}^{2}$ e $\sqrt{E M Q}$ versus as $(\mathrm{p}-1)$ variáveis explicativas para os dois conjunto de variáveis dependentes em que $R_{a j}^{2}$ foi maior, até o máximo de 8 variáveis, sendo que em todos os casos, a variável "espalhamento volumétrico" foi mantida. A escolha de no máximo 8 variáveis explicativas foi para atender a recomendação usual de que para cada variável explicativa no modelo, deve existir em torno de 6 a 10 observações para modelagem. Como neste estudo trabalhou-se com 55 observações, o máximo permitido são 8 variáveis. Escolheu-se o modelo de regressão com 4 parâmetros pois, após a inclusão do quarto parâmetro, a curva do coeficiente de determinação ajustado e o erro médio quadrático dos resíduos se estabilizam (Figura 2).

As variáveis selecionadas, além do espalhamento volumétrico $\left(P_{v}\right)$, foram: índice de biomassa ( $B M I$ ), espalhamento superficial $\left(P_{S}\right)$, helicidade do primeiro mecanismo de espalhamento $\left(\tau_{1}\right)$ e magnitude do tipo de espalhamento do terceiro mecanismo $\left(\alpha_{s 3}\right)$.

Figura 2 - Coeficiente de determinação ajustado $\left(R_{a j}^{2}\right)$ e raiz do erro médio quadrático $(\sqrt{E M Q})$ dos subconjuntos das variáveis explicativas que apresentaram o melhor desempenho com o atributo espalhamento volumétrico incluso.

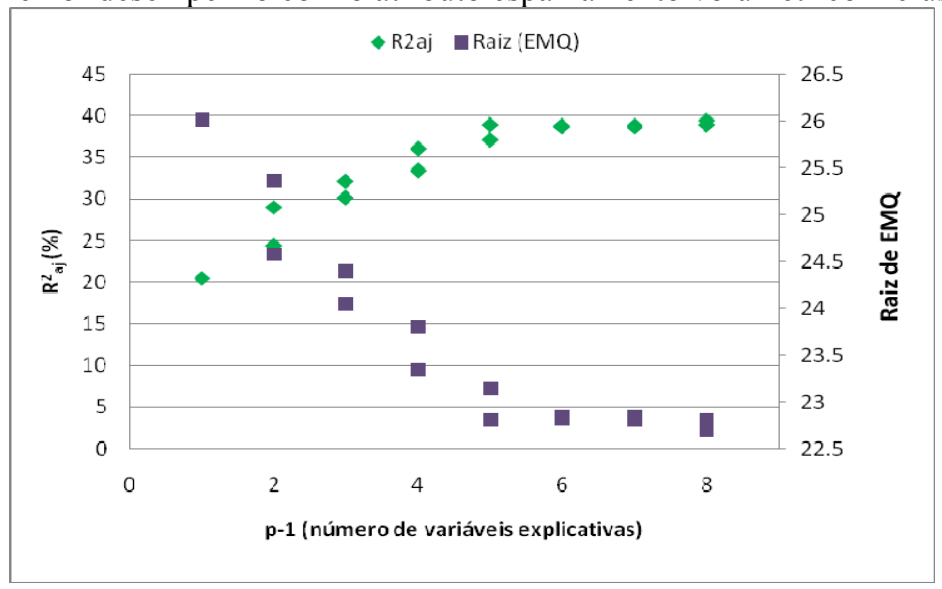


3. Diagnósticos

a) Normalidade da variável dependente. Selecionadas as variáveis explicativas, foi realizada a transformação Box-Cox (Figura 3), a qual normaliza a variável dependente em função das explicativas e, consequentemente, aumenta a correlação entre elas. No eixo y da transformação é mostrada a soma dos quadrados dos resíduos e em x as diferentes estimativas de lambda. Aplicando o teste Shapiro-Wilk, verificou-se que a variável dependente não tinha distribuição Normal, no nível de significância de $5 \%$. A transformação Box-Cox indicou que utilizando um valor de $\lambda$ contido no intervalo $[-0,15,0,70]$, a variável morfológica "volume da haste" passaria a ter distribuição Normal (Figura 3), sendo a estimativa de máxima verossimilhança de lambda (valor estimado de lambda para o qual a soma dos quadrados dos resíduos é mínima) igual a $0,32\left(\chi^{2}=19,43 ; p=0,00001\right)$.

Figura 3 - Comportamento da variável morfológica volume considerando 55 observações e as cinco variáveis explicativas selecionadas.

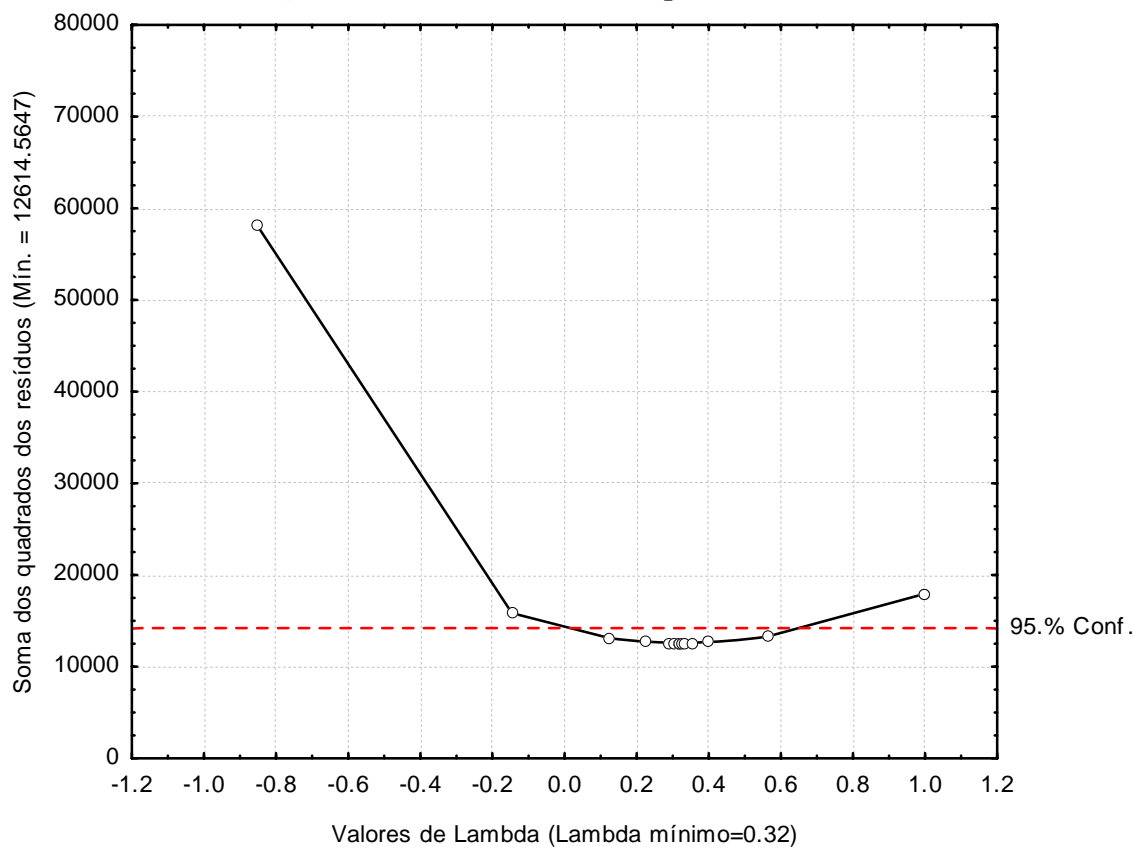

Ao invés de se utilizar o lambda 0,32, utilizou-se a transformação raiz quadrada, a qual é aproximadamente igual ao lambda sugerido e também está contida no intervalo de $95 \%$ de confiança. 
b) Análise exploratória. A Figura 04 apresenta o box-plot da variável dependente "volume da haste" medido em campo $(Y)$ e os diagramas de dispersão entre essa variável $Y$ e cada atributo da imagem ( $\left.X_{1}, \ldots, X p-1\right)$, com o respectivo valor do coeficiente de correlação linear $(r)$. As correlações significativas $(\alpha=0,10)$ são indicadas por um asterisco (*) ao lado do valor de $r$.

Figura 4 - Box-plot da variável dependente (raiz do volume da haste); diagramas de espalhamento e coeficiente de correlação linear entre a variável dependente e cada variável explicativa.

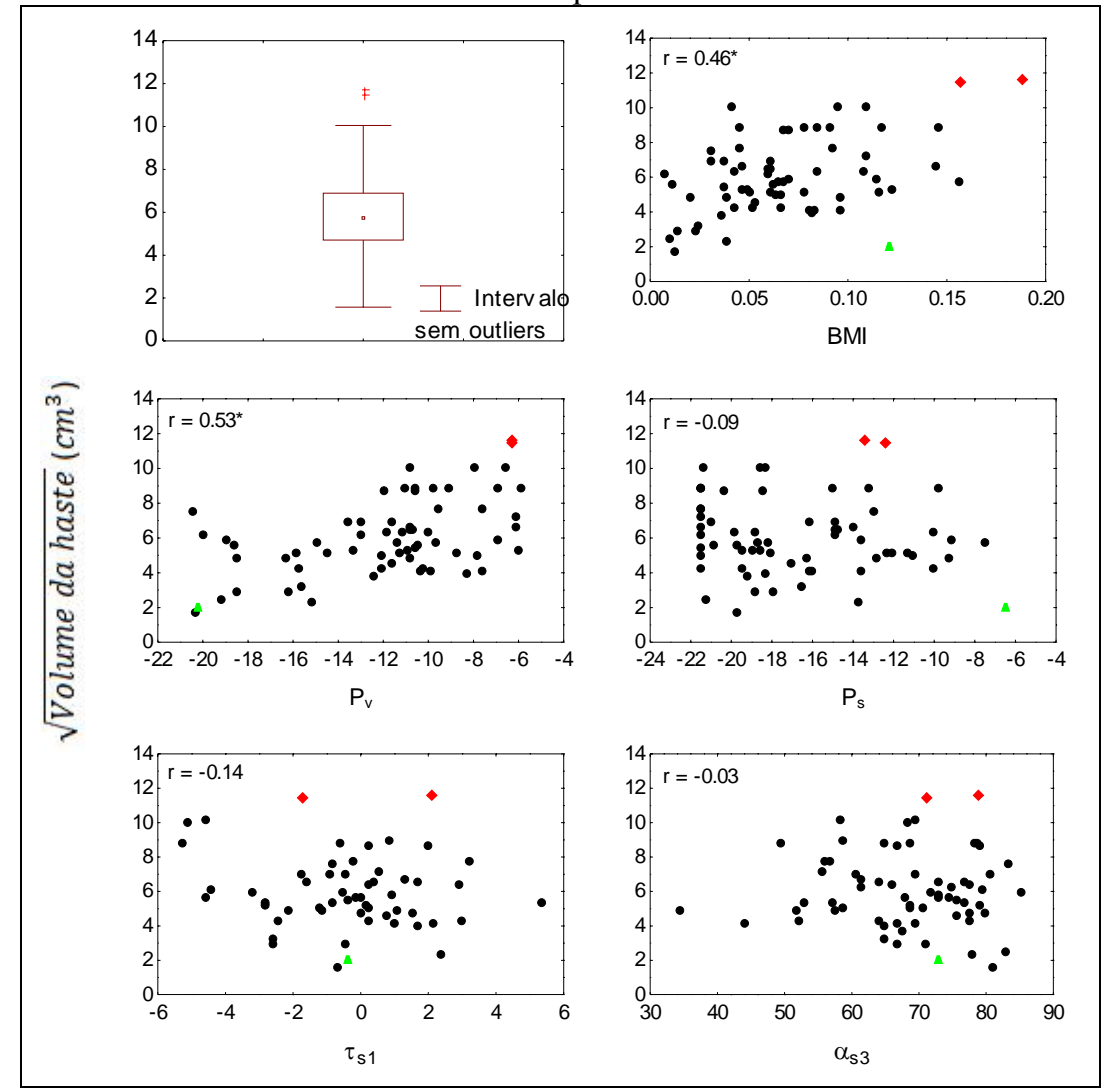

c) Normalidade e homocedasticidade dos resíduos. A Figura 5 mostra o diagrama de dispersão entre o resíduo e o volume da haste no modo 
ajustado (Figura 5a) e o gráfico de probabilidade normal dos resíduos (Figura 5b).

Os resíduos estão distribuídos aleatoriamente (Figura 5a), sem demonstrar um comportamento definido, o que indica que há homocedasticidade. Isso é confirmado pelo teste Levene modificado aplicado para todas as variáveis integrantes do modelo ao nível de significância $5 \% \quad(p(B M I)=0,67$; $\left.p\left(P_{v}\right)=0,48 ; \quad p\left(P_{S}\right)=0,69 ; \quad p(\tau 1)=0,36 ; \quad p\left(\alpha_{s 3}\right)=0,86\right)$.

O padrão de dispersão é aproximadamente linear (Figura $5 \mathrm{~b}$ ), sendo que o teste Shapiro-Wilk confirma a distribuição normal dos resíduos ao nível de significância $5 \%(\mathrm{~S}-\mathrm{W}=0,97$ com $\mathrm{p}$-valor $=0,15)$.

Uma das observações (valor de resíduo próximo de 4 na Figura 5a) foi eliminada da modelagem devido ao seu alto valor de resíduo. Após eliminá-la, os resíduos continuaram homocedásticos $\left(p(B M I)=0,4 ; \quad p\left(P_{v}\right)=0,26\right.$; $\left.p\left(P_{S}\right)=0,58 ; p\left({ }_{\tau 1}\right)=0,59 ; p\left(\alpha_{S 3}\right)=0,84\right)$ e com distribuição normal ao nível de significância $5 \%(\mathrm{~S}-\mathrm{W}=0,96$ com $\mathrm{p}$-valor $=0,1)$.

Figura 5 - Gráficos de resíduos.

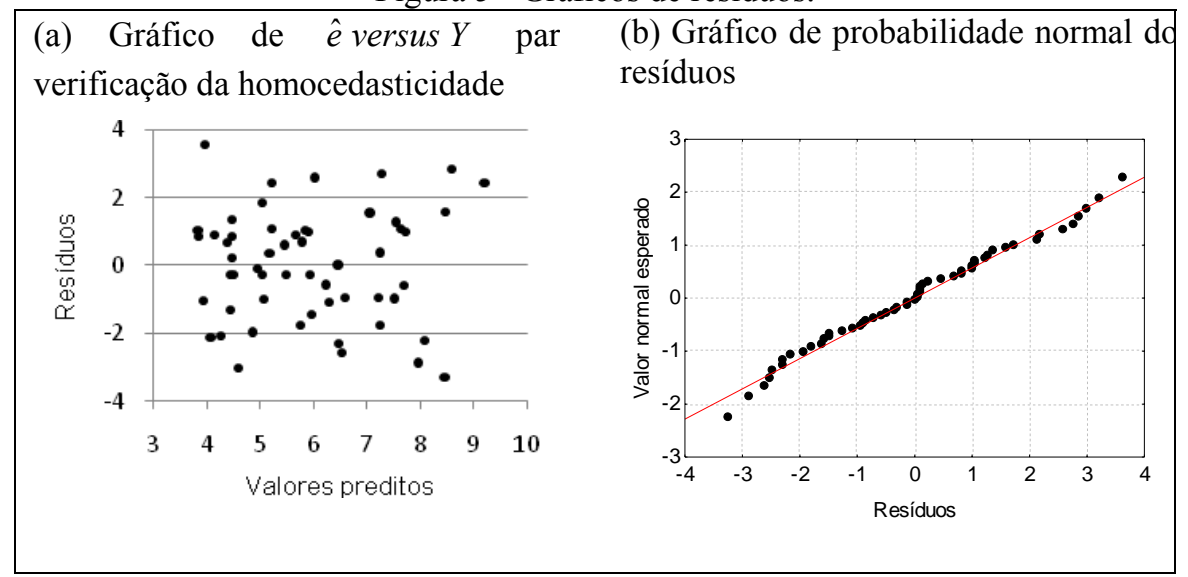

d) Outliers influentes. Podem ser detectados pelas medidas D de Cook ou DFITTS. Se $F($ D.Cook; $6 ; 48)>10 \%$ ou $20 \%$ o outlier é considerado influente. Neste estudo, os dois casos mais influentes apresentaram $F($ D.Cook;6;48) $=1,5 \%$ e $1 \%$. Também, pode ser considerado como outlier se $\mid$ DFFITS $\mid>1$. Uma das observações apresentou $|D F F I T S|=1$ no limite de tolerância. Como os valores da porcentagem referente à distância Cook e do DFFITS são inferiores ao valor considerado influente, nenhum caso foi eliminado. 
e) Termos de interação. Os resíduos do modelo de regressão foram plotados contra cada termo de interação (Figura 6). A análise visual indica a inexistência de nenhum padrão sistemático, não havendo necessidade de inclusão de nenhum dos termos de interação no modelo.

Figura 6 - Diagrama de dispersão entre os resíduos e cada termo de interação.

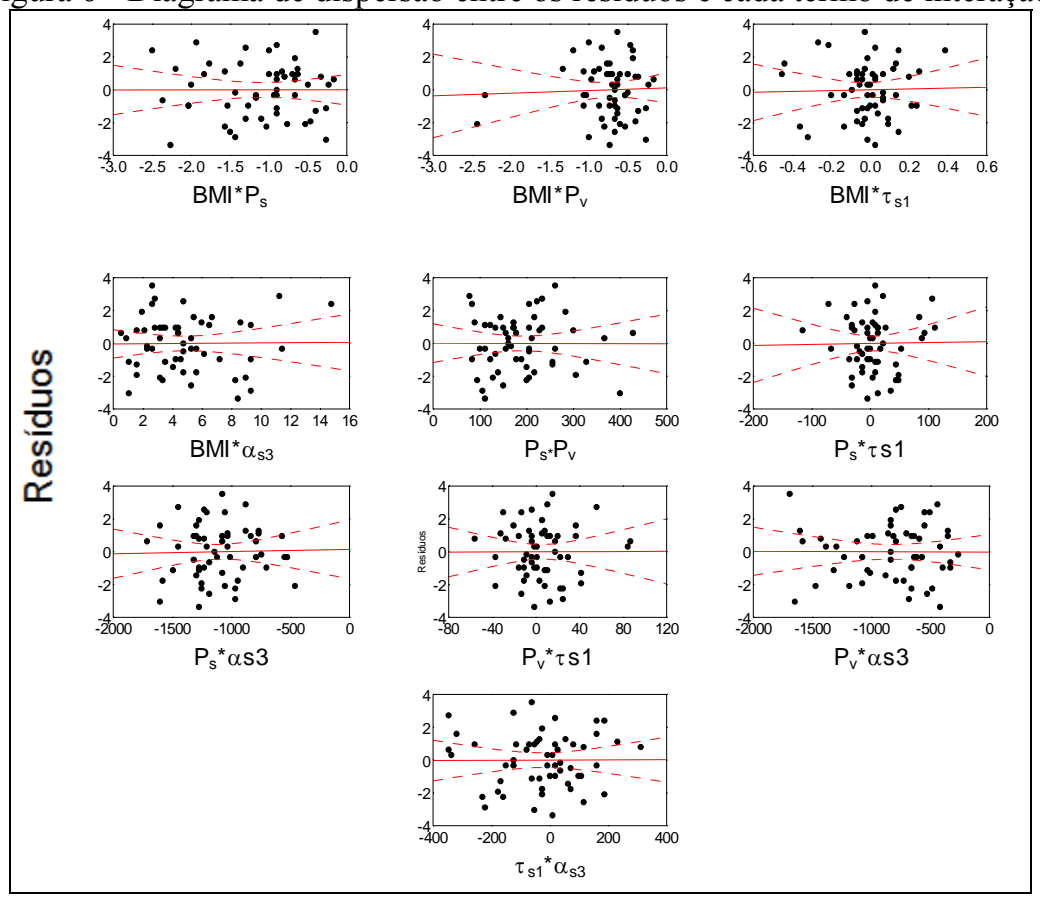

f) Multicolinearidade. O modelo não apresenta colinearidade entre as variáveis, como pode ser observado pelos valores dos fatores de inflação da variância (VIF) (Tabela 1), pois todos são menores que 5 (moderadamente correlacionados).

Tabela 1 - Fatores de inflação da variância.

\begin{tabular}{c|c|c|c|c|c}
\hline Variável & $B M I$ & $P_{S}$ & $P_{v}$ & $\tau_{1}$ & $\alpha_{S 3}$ \\
\hline VIF & 3,7 & 2,3 & 3,6 & 1 & 1,4 \\
\hline
\end{tabular}

g)_Autocorrelação espacial. O diagrama de espalhamento de Moran (Figura 7) mostra que as observações apresentaram resíduos com 
correlação espacial não significativa $(\mathrm{p}=0,26)$ o que indica que o valor do resíduo de uma observação não foi dependente do valor de resíduo nas localizações vizinhas. Há uma pequena tendência a associações espaciais positivas o que significa que uma observação possui vizinhos com valores semelhantes e os vizinhos da observação seriam valores distintos caso a correlação fosse negativa.

Figura 7 - Diagrama de espalhamento de Moran para os resíduos do modelo.

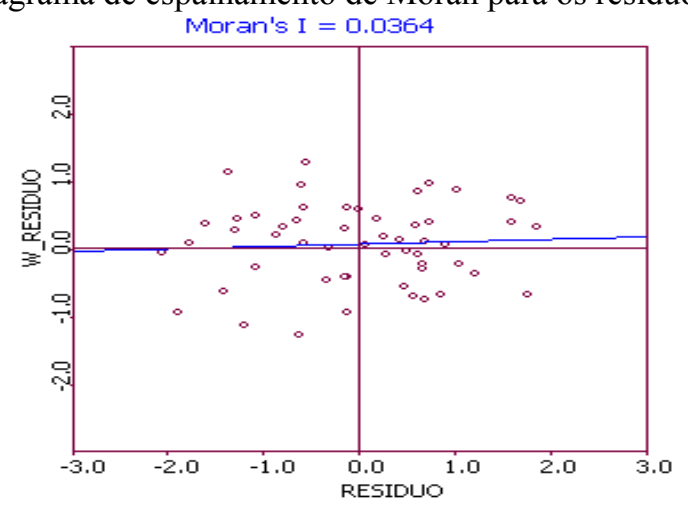

Os diagnósticos indicaram não haver problemas com o modelo de regressão ajustado, dado pela Equação (1):

$$
\sqrt{V}=0,4319+21,4553 B M I \quad 0,1773 P_{S}+0,2189 P_{v} \quad 0,1966 \tau_{1}+0,0638 \alpha_{S 3}
$$

4. Validação. Entre as medidas de avaliação do modelo de regressão (Tabela 2) estão: os coeficientes de regressão padronizados estimados $(\beta)$, os coeficientes de regressão, os respectivos p-valor, o SQR, o MSE, o PRESS e o coeficiente de determinação sem ajuste e com ajuste.

Tabela 2 - Resultado do modelo de regressão para a variável "volume da haste".

\begin{tabular}{c|c|c|c|c|c|c|c|c}
\cline { 2 - 4 } & $\beta$ & $\mathrm{B}$ & $\mathrm{p}$-valor & SQR & MSE & PRESS & $R^{2}$ & $R_{a j}^{2}$ \\
\hline Intercepto & & $-0,432$ & 0,895 & & & & & \\
\hline$B M I$ & 0,4 & 21,455 & 0,049 & & & & & \\
\hline$P_{S}$ & $-0,341$ & $-0,177$ & 0,035 & & & & & \multirow{2}{*}{130,201} \\
\hline$P_{v}$ & 0,407 & 0,219 & 0,042 & & 166,729 & $49 \%$ & $44 \%$ \\
\hline$\tau_{1}$ & $-0,199$ & $-0,197$ & 0,063 & & & & & \\
\hline$\alpha_{S 3} 3$ & 0,307 & 0,064 & 0,017 & & & & & \\
\hline
\end{tabular}


A avaliação do teste $t$ mostrou que as variáveis selecionadas têm importância significativa ao nível de significância de 10\% (todos apresentaram p-valor menor que $7 \%$ ).

O valor de PRESS $(166,729)$ é $28 \%$ maior que o SQR $(130,201)$. Como esta diferença é pequena, o MSE pode ser considerado uma boa estimativa do desvio padrão, ou seja, o MSE correspondente a 2,713 representa um erro de predição de $1,647 \sqrt{\mathrm{cm}^{3}}$. Para a variável estimada média $\left(6,4 \sqrt{\mathrm{cm}^{3}}\right)$, esse erro corresponde a $25 \%$ e para a variável estimada máxima $\left(11,65 \sqrt{\mathrm{cm}^{3}}\right)$ corresponde a $14 \%$.

Apesar do baixo valor do coeficiente de determinação ajustado $\left(R^{2}\right)$, os 10 elementos amostrais reservados para a validação do modelo (Figura 8) ficaram dentro do intervalo de predição de $95 \%$ de confiança.

Figura 8 - Valores de volume observados e estimados para 6 elementos de $P$. repens (1 a 6), 2 de P. elephantipes (7 e 8) e 2 de H. amplexicaulis (8 e 10). A barra vertical corresponde ao intervalo de predição com nível de confiança de $95 \%$.

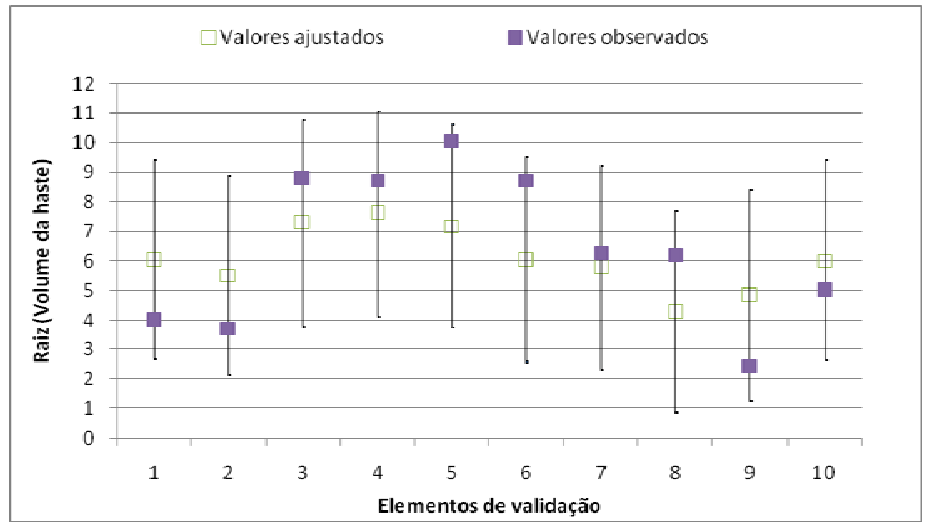

\section{CONCLUSÃO}

Este trabalho permitiu verificar que dentre as cinco variáveis independentes do modelo, quatro foram geradas a partir da decomposição de alvos, sendo duas provenientes da decomposição de Freeman-Durden e duas da decomposição de Touzi. A quinta variável é o parâmetro de Pope índice de biomassa. A construção do modelo usando quatro variáveis geradas a partir da informação de fase do produtoradar mostra a importância deste tipo de informação. Por conseguinte, imagens de radar polarimétricas na banda $\mathrm{L}$ são capazes de fornecer informações relevantes 
para inferência de parâmetros morfológicos de macrófitas da Planície de Inundação Amazônica.

Apesar do modelo não ter apresentado um elevado coeficiente de determinação (44\%), o seu desempenho apresentou boa capacidade preditiva, uma vez que todos os elementos de validação caíram dentro do intervalo de predição de $95 \%$ de confiança. Conclui-se, então, que o modelo pode ser utilizado para a estimativa do volume da haste a partir de dados-radar de banda L e, consequentemente, para a determinação de biomassa, auxiliando, assim, no monitoramento e estimativa da contribuição das macrófitas no ciclo global do carbono.

\section{AGRADECIMENTOS:}

Os autores agradem à FAPESP (Fundação de Amparo à Pesquisa do Estado de São Paulo) (processo: 2008/07537-1), ao PROCAD/CAPES (Programa Nacional de Cooperação Acadêmica/Coordenação de Aperfeiçoamento de Pessoal de Nível Superior) (processo: 0258059), à Dr. Laura Hess por fornecer a imagem PALSAR através do ALOS Kyoto \& Carbon Initiative, à Prof. Chieno Suemitsu (UFOPA) e à sua aluna Ana Sofia S. de Holanda pela ajuda durante o trabalho de campo.

\section{REFERÊNCIAS BIBLIOGRÁFICAS}

ANSELIN, L. The Moran scatterplot as ESDA tool to assess local instability in spatial association. In: FISHER, M. et al. Spatial analytical perspectives on GIS. London: Taylor and Francis, 1996. p. 111-126.

BARTLETT, K.B., CRILL, P.M., BONASSI, J.A., RICHEY, J.E., HARRISS, R.C. Methane flux from the Amazon River floodplain - emissions during rising water. Journal of Geophysical Research, v 95, n. D10, p. 16773-16788, sep/90.

CLOUDE, S.R.; POTTIER, E. A review of target decomposition theorems in radar polarimetry, IEEE Trans Geosc. and Remote Sensing, v. 34, pp. 498-518, 1996.

COSTA, M.P. Estimate of net primary productivity of aquatic vegetation of the Amazon floodplain using Radarsat and JERS-1. International Journal of Remote Sensing, v. 26, n. 20, pp. 4527-4536, 2005.

DEVOL, A.H., RICHEY, J.E., CLARK, W.A., KING, S.L., MARTINELL, L.A. Methane emissions to the troposphere from the Amazon floodplain. Journal of Geophysical Research, v. 93, n. D2, p. 1583-1592. 1988.

FREEMAN, A.; DURDEN, S.L. A three-component scattering model for polarimetric SAR data. IEEE Transactions on Geoscience and Remote Sensing, v. 36, no. 3, pp. 963-973, 1998.

GONÇALVES, F.G.; SANTOS, J.R.; TREUHAFT, R.N. Stem volume of tropical forests from polarimetric radar, International Journal of Remote Sensing, v. 32, n. 2, pp. 503-522, jan/2011.

HENDERSON, F.M.; LEWIS, A.J. Manual of remote sensing: principles and applications of imaging radars, 3. ed. New York: John Wiley \& Sons, 1998. 
HESS, L.; MELACK, J.; FILOSO, S. Delineation of inundated area and vegetation along the Amazon floodplain with the SIR-C synthetic aperture radar. IEEE Trans. on Geoscience and Remote Sensing, v. 33, n. 4, pp. 896-904, 1995.

HESS, L.; et al., Dual-season mapping of wetland inundation and vegetation for the central Amazon basin. Remote Sensing of Environment, v. 87, n. 4, pp. $404-$ 428, 2003.

LEE, J.S.; Refined filtering of image noise using local statistics, Comp. Graph. Image Process, v. 15, pp. 380-389, 1981.

LEE, J.S.; POTTIER, E. Polarimetric radar imaging from basics to applications. New York: CRC Press, 2009.

MITSCH, W.J., GOSSELINK, J.G. Wetlands. 3 ed. Hoboken: John Wiley \& Sons, 2000. 920 pp.

NARVAES, I. Avaliação de dados SAR polarimétricos para estimativa de biomassa em diferentes fitofisionomias de florestas tropicais. 2010. Tese (Doutorado em Sensoriamento Remoto) - Instituto Nacional de Pesquisas Espaciais, São José dos Campos.

NETER, J.; KUTNER, M.H.; NACHTSHEIM, C.J.; LI, W. Applied Linear Statistical Models. 5 ed. New York: McGraw-Hill, 2004.

NOVO, E.M.L.M.; COSTA, M.P.F.; MANTOVANI, J.E.; LIMA, I.B.T. Relationship between macrophyte stand variables and radar backscatter at L and $\mathrm{C}$ band, Tucuruí reservoir, Brazil. Int. J. Remote Sensing, v.23, n.7, pp. 1241-1260, 2002.

PEDRALLI, G. Macrófitas aquáticas como bioindicadoras da qualidade da água: alternativas para usos múltiplos de reservatórios. In: THOMAZ, S. M.; BINI, L. M. Ecologia e manejo de macrófitas aquáticas. Maringá: UEM, 2003, cap.8.

POPE, K.O.; REY-BENAYAS, J.M.; PARIS, J.F. Radar remote sensing of forest and wetland ecosystems in the Central American tropics. Remote Sensing of Environment, v. 48, n. 2, pp. 205-219, 1994.

SARTORI, L.R.; IMAI, N.N.; MURA, J.C.; NOVO, E.M.L.M.; SILVA, T.S.F. Mapping macrophyte species in the Amazon floodplain wetlands using fully polarimetric ALOS/PALSR data. IEEE Transactions on Geoscience and Remote Sensing, v. 49, n. 12, pp. 4717-4728, 2011.

SHAPIRO, S.S. et al. A comparative study of various tests of normality. Journal of the American Statistical Association, v. 63, p. 1343-1372, 1968.

SILVA, T.S.F.; COSTA, M.P.F.; MELACK, J.M. Annual net primary productivity of macrophytes in the Eastern Amazon floodplain, Wetlands, v. 29, n. 2, pp. 747-758, 2009.

SILVA, T.S.F.; COSTA, M.P.F.; MELACK, J.M. Spatial and temporal variability of macrophyte cover and productivity in the eastern Amazon floodplain: A remote sensing approach, Remote Sensing of Environment, v. 114, p. 19982010, 2010. 
THOMAZ, S.M.; BINI, L.M. Análise crítica dos estudos sobre macrófitas aquáticas desenvolvidos no Brasil. In: Ecologia e manejo de macrófitas aquáticas. Maringá: Univ. Estadual de Maringá, 2003. p.19-38.

TOUZI, R. Target scattering decomposition in terms of roll-invariant target parameters, IEEE Transactions on Geoscience and Remote Sensing, v. 45, n. 1, pp. 73-84, 2007.

VASCONCELOS, C.H.; NOVO, E.M.L.M; DONALISIO, M.R. Uso do sensoriamento remoto para estudar a influência de alterações ambientais na distribuição da malária na Amazônia brasileira. Cadernos de Saúde Pública. v. 22, pp. 517-526, 2006.

(Recebido em novembro de 2011. Aceito em março de 2012). 